\title{
Electrical Characterization of Traps in AlGaN/GaN FAT-HEMT's on Silicon Substrate by C-V and DLTS Measurements
}

\author{
Manel Charfeddine ${ }^{1}$, Malek Gassoumi ${ }^{1}$, Hana Mosbahi ${ }^{1}$, Christophe Gaquiére ${ }^{2}$, \\ Mohamed Ali Zaidi ${ }^{*}$, Hassen Maaref ${ }^{1}$ \\ ${ }^{1}$ Laboratoire des Micro-optoéLectroniques et Nanostructures, Université de Monastir, Faculté des \\ Sciences de Monastir, Monastir, Tunisie \\ ${ }^{2}$ Institut d'Electronique de Microélectronique et de Nanotechnologie IMEN, Département Hyperfréquences \\ et Semiconducteurs, Université des Sciences et Technologies de Lille, \\ Villeneuve d'Ascq Cedex, France \\ E-mail: ${ }^{*}$ MAli.Zaidi@fsm.rnu.tn
}

Received June 8, 2011; revised August 2, 2011; accepted August 13, 2011

\begin{abstract}
We investigate high electron mobility transistors (HEMT's) based on AlGaN/GaN grown by molecular beam epitaxy on Silicon substrates. The improvement of the performances of such transistors is still subject to the influence of threading dislocations and point defects which are commonly observed in these devices. Deep levels in FAT-HEMT's are characterized by using Capacitance-Voltage $(\mathrm{C}-\mathrm{V})$ measurements, from which we can extract the barrier height and the donor concentration in the AlGaN layer. Deep Level Transient Spectroscopy (DLTS) Technique is also employed to identify defects in the heterostructure. Measurements reveal the presence of one electron trap with the activation energy $E_{1}=0.30 \mathrm{eV}$ and capture cross-section $\sigma_{n}$ $=3.59 \times 10^{-19} \mathrm{~cm}^{2}$. The localization and the identification of this trap have been discussed.
\end{abstract}

Keywords: AlGaN/GaN, FAT-HEMT’s, C-V, Capacitance DLTS, Deep Level

\section{Introduction}

The material semiconductor GaN nitride and associated alloys (III-N compounds) arouse since more than three decades a regained interest because of their properties and their exceptional robustness [1-3]. These large gap materials, which extend from 0.7 to $6.1 \mathrm{eV}$, allow the realization of heterostructures in order of applications in the field of the ultra high frequencies (RF) power components $[4,5]$. A new market thus emerges in the field of telecommunications with High Electronic Mobility Transistors (HEMT's) which can support power densities 10 times higher than those accessible with the silicon and gallium arsenide technologies as reported by Dumka et al. [6], as well as cut-off frequencies higher than 100 $\mathrm{GHz}$ were reported in a previous paper [7]. These HEMT's structures are essential to meet the increasing needs for the communication systems requiring high power and frequency applications [8-10] (radars, stations of bases, connection satellite). Due to their strong ther- mal conductivity and good performance stability in a hostile environment, GaN-based HEMT devices are excellent candidates especially in high power/frequencies domain. Thus, many applications turn to the GaN-based components, in addition to RF power amplifiers $[11,12]$.

Silicon substrate presents a significant attraction from its very competitive price, the size of the substrates available (up to 12 "). It has a good thermal conductivity close to the one of GaN. In addition, the (111) orientation with a 6-fold symmetry is preferred for the GaNbased heterostructures on the silicon substrate [13]. The development of GaN-based devices on silicon mostly relies on the control of material quality, espe- cially the management of the dislocation density and of the stress due to the thermal expansion coefficient mis- match between GaN and silicon. Thus, Silicon presents a certain number of determining advantages for the mass production of III-Nitride devices. The realization of powerful and reliable FAT-HEMT transistors III-N requires a buffer layer with good electric insulation proprieties, in 
addition to an electrical substrate insulator with a good thermal conductivity [14-16]. In FAT-HEMT $(\mathrm{Al}, \mathrm{Ga}) \mathrm{N} /$ $\mathrm{GaN}$ heterostructures. The compensation of the residual doping of type $\mathrm{N}$ of the buffer layers is generally ensured by intrinsic mechanisms related to defects.

Although the significant progress obtained on the processes of development, few studies were devoted to the electric defect characterization, and the mechanisms of electric conduction in the devices. To this end, research works have been undertaken in the subject of trapping effects with the use of different electrical characterization techniques, including photoionization spectroscopy [17], drain leakage-current measurements [18], current-deep level transient spectroscopy (CDLTS) [19] and deep level transient spectroscopy (DLTS) [20], Current-Voltage, I(V) [21] and Capacitance-Voltage C(V) measurements [22].

In this paper, we intend to analyze the $\mathrm{C}(\mathrm{V})$ characteristics of FAT-HEMT AlGaN/GaN transistors based on $\mathrm{Si}$ substrate. Then we will characterize the electrical active defects present in the structure, using DLTS. This technique has been proved to be a powerful tool to probe the electronic properties of FAT-HEMT AlGaN/GaN transistors. The localization and the identification of these traps are discussed.

\section{Sample Description}

The investigated structure consists of an $\mathrm{AlGaN} / \mathrm{GaN}$ FAT-HEMT's grown on resistive silicon (111) substrate (4000 - $10.000 \Omega \mathrm{cm}$ ) using ammonia (Riber Compact 21). The epitaxial layers are obtained by molecular beam epitaxy (MBE). Details on the epitaxial growth are reported elsewhere [23] and layer ticknesses are taken as follows: a $100 \mathrm{~nm}$ AlN nucleation layer is at first grown on the $\mathrm{Si}$ (111) substrate followed by a $2 \mu \mathrm{m}$ thick GaN and a $30 \mathrm{~nm}$-thick undoped $\mathrm{AlGaN}$ layer encapsulated with $1 \mathrm{~nm}$ of an unintentionally doped (UID) GaN cap layer. This cap layer is grown to ensure the ohmic contact. The ohmic metallization contacts were prepared by evaporating $\mathrm{Ti} / \mathrm{Al} / \mathrm{Ni} / \mathrm{Au}$ multilayer and rapid-thermal annealing at $850^{\circ} \mathrm{C}$ in a $\mathrm{N}_{2}$ atmosphere for $30 \mathrm{~s}$. For the gate contact, a thick $\mathrm{Ni}$ layer covered by $\mathrm{Au}$ layer $(\mathrm{Ni} / \mathrm{Au})$ and patterned by e-beam lithography is employed with a Schottky area of $250 \times 200 \mu \mathrm{m}^{2}$.

Deep level transient spectroscopy (DLTS) is one of the most versatile techniques used to determine the electrical properties of defects. For that, it has been used as a technique to characterize the electron traps in the $\mathrm{AlGaN} / \mathrm{GaN} / \mathrm{Si}$ heterostructures. Measurements were performed using double lock-in detection and a PAR 410 capacitance meter with a frequency of $1 \mathrm{MHz}$ recorded in the temperature range $10-320 \mathrm{~K}$ using Helium cooled cryostat.

\section{Experimental Results and Discussion}

We first briefly discuss the $\mathrm{C}-\mathrm{V}$ results. In order to validate the presence of a two-dimensional electron gas (2-DEG), we took measurements of Capacitance-Voltage $(\mathrm{C}-\mathrm{V})$ using a capacitance meter of $1 \mathrm{MHz}$ frequency (Figure 1). We note that the capacitance variation as a function of the gate voltage shows the existence of a capacitance plateau appearing from $8 \mathrm{~V}$ to $2 \mathrm{~V}$, and it is associated to the depletion of the two-dimensional electron gas (2-DEG), located at the heterointerface. Between the plateau and the right part, there is a transition region, where the capacitance decreases rapidly with decreasing applied voltage. Then, we observe a sharp fall of the capacitance at the bias known as the pinch-off voltage $(V p=0.5 \mathrm{~V})$. The region beyond the $\mathrm{V}_{\mathrm{p}}$ voltage is named the residual capacitance region. This is a characteristic of the 2-DEG. C(V) measurements allow estimating the values of the applied pulsate with voltage and the reverse bias in DLTS measurements

The C-V characteristics of the AlGaN/GaN/Si FATHEMT's measured at different temperatures are presented in Figure 2. It shows good reproducibility even when a forward voltage of $0.5 \mathrm{~V}$ is applied.

This figure reveals that the capacitance decreases when increasing the temperature. The maximal value of the capacitance ranges from 0.82 to $1.1 \mathrm{nF}$. When the temperature increases, the pinch-off voltage position shifts towards the high voltage. The pinch-off voltage shift at $300 \mathrm{~K}$ is more gradual than that at $100 \mathrm{~K}$. This significant shift informs us about the presence of the defects which are probably localized at the interface and in the vicinity of the accumulation region. The defects are

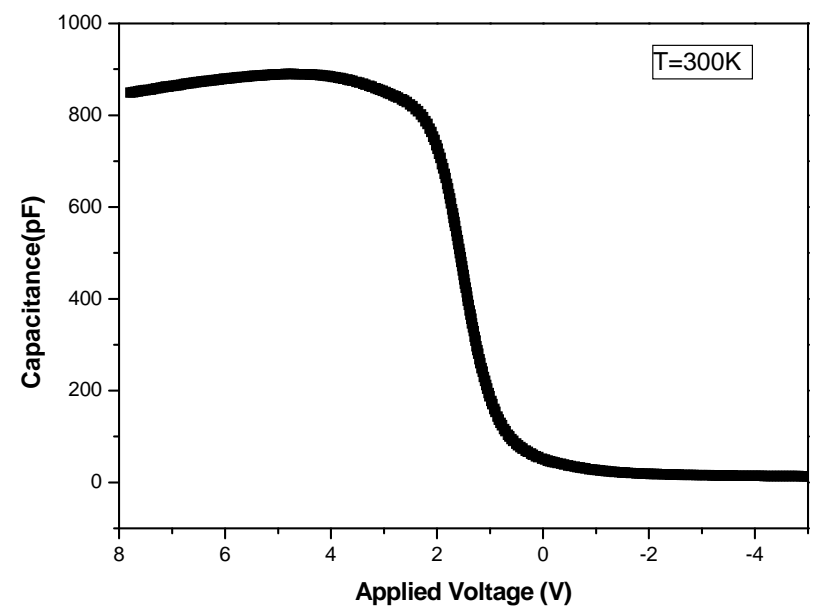

Figure 1. Capacitance-Voltage measurements results obtained on AlGaN/GaN/Si FAT-HEMTs at $T=300 \mathrm{~K}$. 


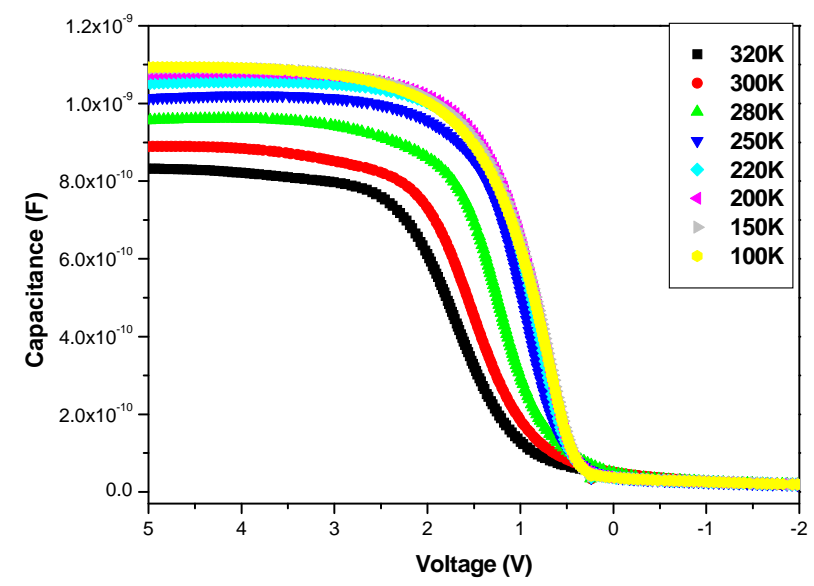

Figure 2. The C-V characteristics of the AlGaN/GaN/Si FAT-HEMTs measured at temperatures ranging from 100 K to $320 \mathrm{~K}$.

present probably at the same range of temperatures. This will be confirmed by using DLTS technique.

We have deduced the carrier concentration profile $N_{c-v}$ versus the space charge depth $W$ in the heterostructure according to the following relation:

$$
N_{C-V}=\frac{C^{3}}{q \varepsilon S^{2}(d C / d V)}
$$

and

$$
W=S \frac{\varepsilon_{0} \varepsilon_{r}}{C}
$$

where $S$ is the surface of the Schottky contact $\varepsilon_{0}$ is the free-space dielectric constant $\varepsilon_{r}$ is the relative dielectric constant of AlGaN barrier and $\mathrm{q}$ is the elementary charge. The results are plotted in Figure 3. It exhibits a strong peak of a carrier density equals to $1.60 \times 10^{21}$ $\mathrm{cm}^{-3}$ corresponding to the presence of a gas (2DEG) at the interface $\mathrm{AlGaN} / \mathrm{GaN}$. The position of this peak proves that AlGaN layer thickness is about $32 \mathrm{~nm}$. In addition, we have determined the net doping concentration $N_{D}$ and the barrier height $\varphi_{B}$ from the lot of $1 / C^{2}$ as a function of gate voltage (Figure 4). We have found $N_{D}=$ $4.4010^{19} \mathrm{~cm}^{-3}$ and $\varphi_{B}=0.9 \mathrm{eV}$.

To detect, locate and identify the localization of deep traps in FAT-HEMT transistors, we have carried out deep levels transient spectroscopy measurements for a reverse applied voltage of $2 \mathrm{~V}$ with an amplitude of $2 \mathrm{~V}$, a filling time $\left(t_{p}\right)$ was fixed to $0.5 \mathrm{~ms}$ and for different emission rate.

Typical DLTS capacitance spectra (Figure 5) of Al$\mathrm{GaN} / \mathrm{GaN} / \mathrm{Si}$ HEMTs at $\mathrm{V}_{\mathrm{gs}}=-2 \mathrm{~V}$ reveal the presence of only one peak corresponding to electron emission from one trap named $E_{1}$, The apparent activation energy $\mathrm{E}_{\mathrm{a}}$ and the capture cross-section $\sigma_{n}$ associated to the ob-

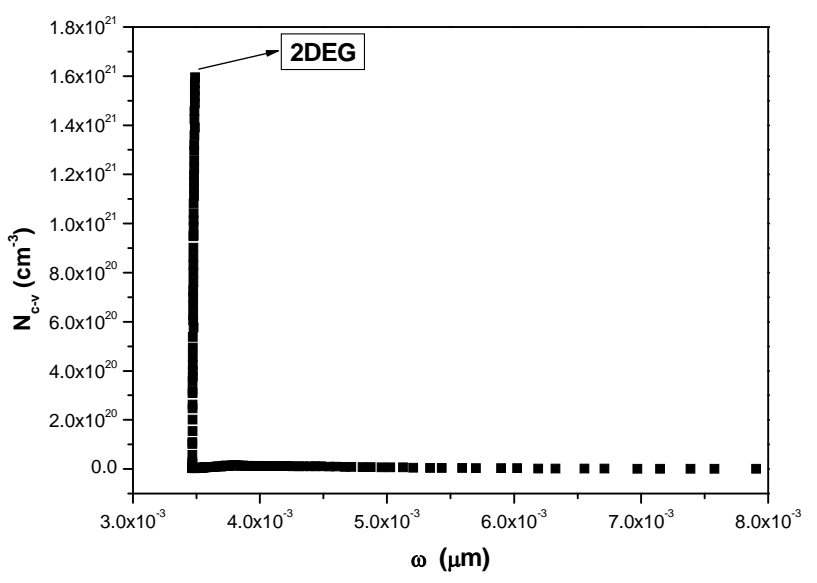

Figure 3. Carrier distribution profile.

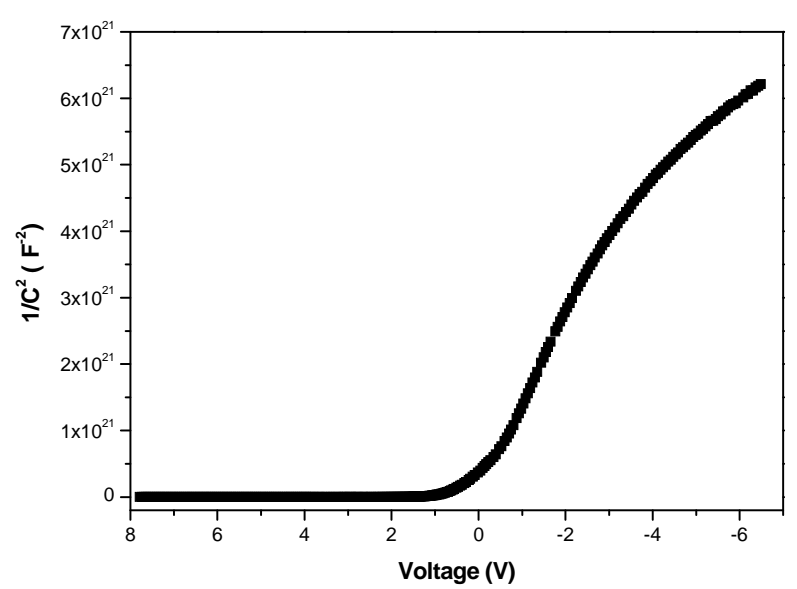

Figure 4. Curves represent $1 / C^{2}$ according to Voltage.

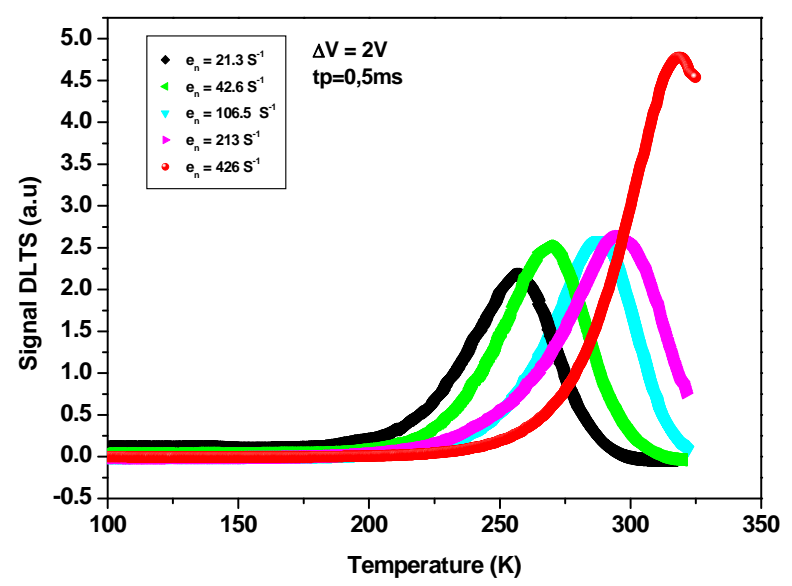

Figure 5. A typical capacitance DLTS spectrum showing the presence of one level performed on AlGaN/GaN/Si FAT-HEMTs. Recording condition: different emission rate, reverse bias $2 \mathrm{~V}$, and filling time $t_{p}=0.5 \mathrm{~ms}$.

served electron trap are extracted from the Arrhenius plot of: $\operatorname{Ln}\left(T^{2} / e_{n}\right)$ versus $1000 / T$. The electrons are emitted as a consequence of thermal activation. The thermal emis- 
sion rate $e_{n}$ is written as [24]:

$$
e_{n}=\delta_{n} T^{2} \sigma \exp \left(\frac{-E_{a}}{K T}\right)
$$

with

$$
\delta_{n}=\frac{4 \Pi \sqrt{6} \Pi^{3 / 2} K_{\beta}^{2} m_{n}^{*}}{h^{3}}
$$

This relation applied to the peak temperatures at which the electron emission rate, $\mathrm{e}_{\mathrm{n}}$, equals the rate window setting. In Equation (2), where $m_{n}^{*}=m_{0} m_{G a N}$ such as $m_{n}^{*}$ is the electron effective mass , $\mathrm{m}_{0}$ is the mass of the free electron and $m_{G a N}=0.22$ is the relative electrons mass in the GaN case, $E_{a}$ the activation energy, $\sigma_{n}$ the capture cross section, the temperature, $h$ and $k_{\beta}$ are the Planck and Boltzmann constant respectively. The capture cross section was assumed to be independent of temperature and the value of $m_{n}^{*}$. This Arrhenius plot allows an identification of a deep level defect. The results are presented in Figure 6.

The electron trap $E_{1}$, peaked at $T=318 \mathrm{~K}$ has an activation energy $E_{a}=(0.30 \pm 0.02) \mathrm{eV}$ and a capture cross-section $\sigma_{n}=3.59 \times 10^{-19} \mathrm{~cm}^{2}$. This $\mathrm{E}_{\mathrm{a}}$ value was previously found by Sghaier et al. [25] observed a similar defect with an activation energy close to $0.31 \mathrm{eV}$ by DLTS using boxcar technique. Nozaki et al. [26] have shown a comparable defect with an activation energy of $0.28 \mathrm{eV}$ using I-DLTS (current-DLTS) technique performed on AlGaN/GaN MODFET. They attribute this defect to dislocation localized in the AlGaN layer. This defect was also studied by Tang [27], with a sample growth of unintentionally doped (UID) semi-insulating $\mathrm{GaN}$ on $\mathrm{SiC}$ which is mainly employed in GaN HEMT devices. To investigate the defect proprieties and their signatures, they used the PL technique correlated with a typical net TSC electric technique. They obtained an activation energy $E a=0.30 \mathrm{eV}$. This value is in good agreement with the value characterizing the defect found in our heterostructure. The deep level, as an extendeddefect, has been also widely observed by DLTS in n-type $\mathrm{GaN}$ and $\mathrm{AlGaN} / \mathrm{GaN}$ heterostructures [28].

The trap carrier concentration is extracted according to the relation (3) with the approximation $N_{T}<N_{D}$ :

$$
\frac{\Delta C}{C_{0}} \approx \frac{1}{2} \frac{N_{T}}{N_{D}}
$$

The carrier concentration obtained is about $N_{T}=6.85 \times$ $10^{16} \mathrm{~cm}^{-3}$ in our sample Si doped. Nevertheless, it should be mentioned that the trap with electrons located at approximately $0.30 \mathrm{eV}$ of the conduction band is present into strong concentration probably due to Si dopage. A similar carrier concentration of this defect is observed by

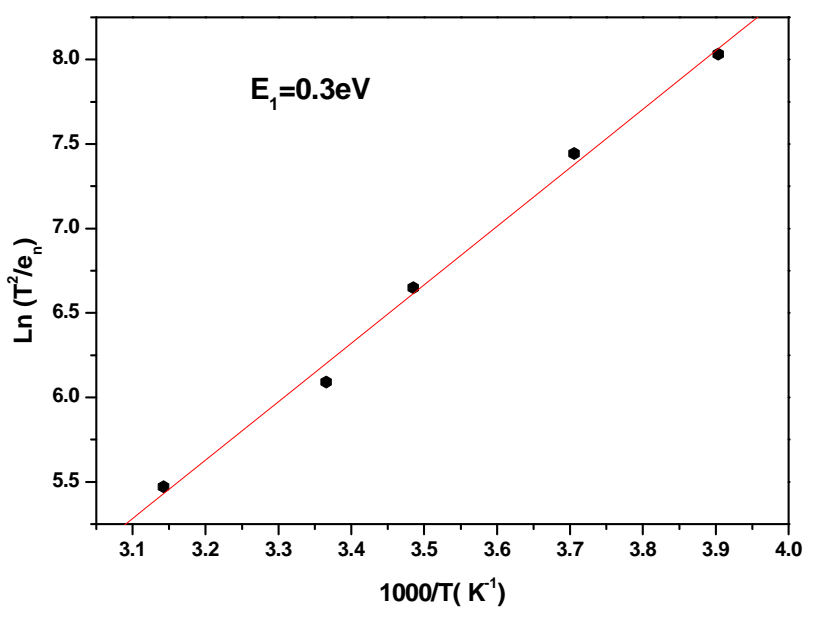

Figure 6. Arrhenius plots for $E_{1}$ deep levels observed in AlGaN/GaN/Si FAT-HEMTs.

Mohamed et al. [29] by using charge deep level transient spectroscopy (Q-DLTS) in the n-GaN sample. Our results seem to be a dislocation extended from buffer layer to the AlGaN layer.

\section{Conclusions}

In summary, we have investigated static measurements and defect analysis on FAT-HEMT AlGaN/GaN realized on $\mathrm{Si}$ substrate grown by MBE. Defects analysis performed on these transistors by $\mathrm{C}-\mathrm{V}$ characteristics and DLTS Signal shows one deep defect that we extracted its signature using on the Arrhenius plot. This trap is attributed to dislocation localized in the AlGaN layer. Finally, the extraction of the deep trap concentration has been evidenced for AlGaN/GaN FAT-HEMTs.

\section{References}

[1] P. Omling, E. R. Weber, L. Montelius, H. Alexander and J. Michel, "Electrical Properties of Dislocations and Point Defects in Plastically Deformed Silicon," Physical Review $B$, Vol. 32, No. 20, 1985, pp. 6571-6581. doi:10.1103/PhysRevB.32.6571

[2] M. A. Khan, A. Bhattarai, J. N. Kuznia and D. T. Olson, "High Electron Mobility Transistor Based on a $\mathrm{GaN}-\mathrm{Al}_{\mathrm{X}}$, $\mathrm{Ga}_{1-\mathrm{X}} \mathrm{N}$ Heterojunction," Applied Physics Letters, Vol. 63, No. 9, 1993, pp. 1214-1215. doi:10.1063/1.109775

[3] L. Semra, A. Teliaa and A. Soltani, "Trap Characterization in AlGaN/GaN HEMT by Analyzing Frequency Dispersion in Capacitance and Conductance," Surface and Interface Analysis, Vol.42, 2010, pp. 799-802. doi:10.1002/sia.3462

[4] H. Kim, S. J. Park and H. Hwang, "Design and Fabrication of Highly Efficient GaN-Based Light-Emitting Diodes," IEEE, Vol. 49, No. 10, 2002, pp. 1715-1721.

[5] R. D. Dupuis, "Epitaxial Growth of III-V Nitride Semi- 
conductors by Metalorganic Chemical Vapor Deposition," Journal of Crystal Growth, Vol. 178, 1997, pp. 56-73. doi:10.1016/S0022-0248(97)00079-1

[6] D. C. Dumka, C. Lee, H. Q. Tserng, P. Saunier and M. Kumar, "AlGaN/GaN HEMTs on Si Substrate with 7 W/mm Output Power Density at $10 \mathrm{GHz}$," Electronics Letters, Vol.40, 2004, pp. 1023-1024. doi:10.1049/el:20045292

[7] A. Minko, V. Hoël, E. Morvan, B. Grimbert, A. Soltani, E. Delos, D. Ducatteau, C.Gaquière, D. Théron, J. C. D. Jaeger, H. Lahreche, L. Wedzikowski, R. Langer and P. Bove, "AlGaN-GaN HEMTs on Si with Power Density Performance of $1.9 \mathrm{~W} / \mathrm{mm}$ at $10 \mathrm{GHz}$," IEEE Electron Device Letters, Vol. 25, 2004, pp. 453-455. doi:10.1109/LED.2004.830272

[8] M. A. Khan, J. N. Kuznia, D. T. Olson, J. M. Van Hove, M. Blasingame and L. F. Reitz, "High-Responsivity photo-Conductive Ultraviolet Sensors Based on Insulating Single-Crystal GaN Epilayers," Applied Physics Letters, Vol. 60, 1992, pp. 2917-2919.

doi: $10.1063 / 1.106819$

[9] S. Sttrite and H. Morkoç, "GaN, AlN, and InN: A Review," Journal of Vacuum Science \& Technology B, Vol. 10, 1992, pp. 1237-1266. doi:10.1116/1.585897

[10] M. A. Khan, J. N. Kuznia, D. T. Olson, M. Blasingame and A. R. Bhattarai, "Schottky Barrier Photodetector Based on $\mathrm{Mg}$ - Doped $p$ - Type GaN Films," Applied Physics Letters, Vol.63, 1993,pp. 2455-2456. doi:10.1063/1.110473

[11] X. L. Wang, T. S. Shen, H. L. Xiao, C. M. Wang, G. X. Hu, W. J. Luo, J. Tang, L. C. Guo and J. M. Li, "HighPerformance $2 \mathrm{~mm}$ Gate Width GaN HEMTs on $6 \mathrm{H}-\mathrm{SiC}$ with Output Power of 22.4 W@ 8 GHz," Solid-State Electron, Vol. 52, 2008, pp. 926-929. doi:10.1016/j.sse.2007.12.014

[12] D. Ducatteau, A. Minko, V. Hoël, E. Morvan, E. Delos, B. Grimbert, H. Lahreche, P. Bove, C. Gaquière, J. C. De Jaeger and S. Delage, "Output Power Density of 5.1/mm at $18 \mathrm{GHz}$ with an AlGaN/GaN HEMT on Si Substrate," IEEE Electron Device Letters, Vol. 27, 2006, pp. 7-9. doi:10.1109/LED.2005.860385

[13] T. Ito, Y. Nomura, S. L. Selvaraj and T. Egawa," Comparison of Electrical Properties in GaN Grown on $\mathrm{Si}(111)$ and c-Sapphire Substrate by MOVPE," Journal of Crystal Growth, Vol. 310, 2008, pp. 4896-4899. doi:10.1016/j.jcrysgro.2008.08.029

[14] N. Maeda, K. Tsubaki, T. Saitoh, T. Tawara and N. Kobayashi, "High-Temperature Electron Transport Properties in AlGaN/GaN Heterostructures," Applied Physics Letters, Vol. 79, 2001, pp. 1634-1636. doi:10.1063/1.1400779

[15] N. Maeda, T. Saitoh, K. Tsubaki, T. Nishida and N. Kobayashi, "Two-Dimensional Electron Gas Transport Properties in AlGaN/GaN Single- and Double-Heterostructure Field Effect Transistors," Materials Science and Engineering: B, Vol. 82, 2001, pp. 232-237. doi:10.1016/S0921-5107(01)00604-3

[16] N. Maeda, T. Nishida, N. Kobayashi and M. Tomizawa,
"Two-Dimensional Electron-Gas Density in $\mathrm{Al}_{X} \mathrm{Ga}_{1-X} \mathrm{~N} /$ GaN Heterostructure Field-Effect Transistors," Applied Physics Letters, Vol. 73, No. 13, 1998, pp. 1856-1858. doi:10.1063/1.122305

[17] P. B. Klein, "Photoionization Spectroscopy in AlGaN/ GaN High Electron Mobility Transistors," Journal of Applied Physics, Vol. 92, 2002, pp. 5498-5502. doi:10.1063/1.1510564

[18] S. Arulkumaran, T. Egawa, H. Ishikawa and T. Jimbo, "Comparative Study of Drain-Current Collapse in AlGaN/GaN High-Electron-Mobility Transistors on Sapphire and Semi-Insulating SiC," Applied Physics Letters, Vol. 81, No. 16, 2002, pp. 3073-3075.

doi: $10.1063 / 1.1512820$

[19] O. Mitrofanov, M. Manfra and N. Weimann, "Impact of Si Doping on Radio Frequency Dispersion in Unpassivated GaN/AlGaN/GaN High-Electron-Mobility Transistors Grown by Plasma-Assisted Molecular-Beam Epitaxy," Applied Physics Letters, Vol. 82, No. 24, 2003, pp. 4361-4363. doi:10.1063/1.1582373

[20] A. P. Zhang, L. B. Rowland, E. B. Kaminsky, V. Tilak, J. C. Grande, J. Teetsov, A. Vertiatchikh and L. F. Eastman, "Correlation of Device Performance and Defects in AlGaN/GaN High-Electron Mobility Transistors," Journal of Electronic Materials, Vol. 32, No. 5, 2003, pp. 388394. doi:10.1007/s11664-003-0163-6

[21] M. Gassoumi, J. M. Bluet, G. Guillot, C. Gaquière and H. Maaref, "Characterization of Deep Levels in High Electron Mobility Transistor by Conductance Deep Level Transient Spectroscopy," Materials Science and Engineering $C$, Vol. 28, 2008, pp. 787-790. doi:10.1016/i.msec.2007.10.068

[22] R. Mosca, E. Gombia, A. Passaseo, V. Tasco, M. Peroni and P. Romanini, "DLTS Characterization of Silicon Nitride Passivated AlGaN/GaN Heterostructures," Superlattices and Microstructures, Vol. 36, 2004, pp. 425-433. doi:10.1016/j.spmi.2004.09.006

[23] V. Hoel, N. Vellas, C. Gaquiere, J. C. DeJaeger, Y. Cordier, F. Semond, F. Natali and J. Massies, "High-Power AlGaN/GaN HEMTs on Resistive Silicon Substrate," Electronics Letters, Vol. 38, 2002, pp. 750-752. doi:10.1049/el:20020522

[24] J. Osaka, Y. Ohno, S. Kishimoto, K. Maezawa and T. Mizutani, "Deep Levels in $n$-Type AlGaN Grown by Hydride Vapor-Phase Epitaxy on Sapphire Characterized by Deep-Level Transient Spectroscopy," Applied Physics Letters, Vol. 87, 2005, pp. 222112-1-222112-3. doi:10.1063/1.2137901

[25] N. Sghaier, M. Trabelsi , N. Yacoubi, J. M. Bluet, A. Souifi, G. Guillot, C. Gaquière and J. C. Dejaeger, "Traps Centers and Deep Defects Contribution in Current Instabilities for AlGaN/GaN HEMT's on Silicon and Sapphire Substrates," Microelectronics Journal, Vol. 37, 2006, pp. 363-370. doi:10.1016/j.mejo.2005.05.014

[26] S. Nozaki, H. Feick, E. R. Weber, M. Micovic and C. Nguyen, "Compression of the dc Drain Current by Electron Trapping in AlGaN/GaN Modulation Doped FieldEffect Transistors," Applied Physics Letters, Vol.78, No. 
19, 2001, pp. 2896-2898. doi:10.1063/1.1367274

[27] H. Tang, Z. Q. Fang, S. Rolfe, J. A. Bardwell and S. Raymond, "Growth Kinetics and Electronic Properties of Unintentionally Doped Semi-Insulating GaN on $\mathrm{SiC}$ and High-Resistivity GaN on Sapphire Grown by Ammonia Molecular-Beam Epitaxy," Journal of Applied Physics, Vol. 107, 2010, pp. 103701-1-103701-12.

[28] Z.-Q. Fang, D. C. Look, D. H. Kim and I. Adesida, "Traps in $\mathrm{AlGaN} / \mathrm{GaN} / \mathrm{SiC}$ Heterostructures Studied by
Deep Level Transient Spectroscopy," Applied Physics Letters, Vol. 87, 2005, pp. 182115-1-182115-3. doi:10.1063/1.2126145

[29] Z. H. Mahmood, A. P. Shah, A. Kadir, M. R.Gokhale, A. Bhattachary and B. M. Arora, "Charge Deep Level Transient Spectroscopy of Electron Traps in MOVPE Grown n-GaN on Sapphire," physica status solidi, Vol. 245, 2008, pp. 2567-2571 